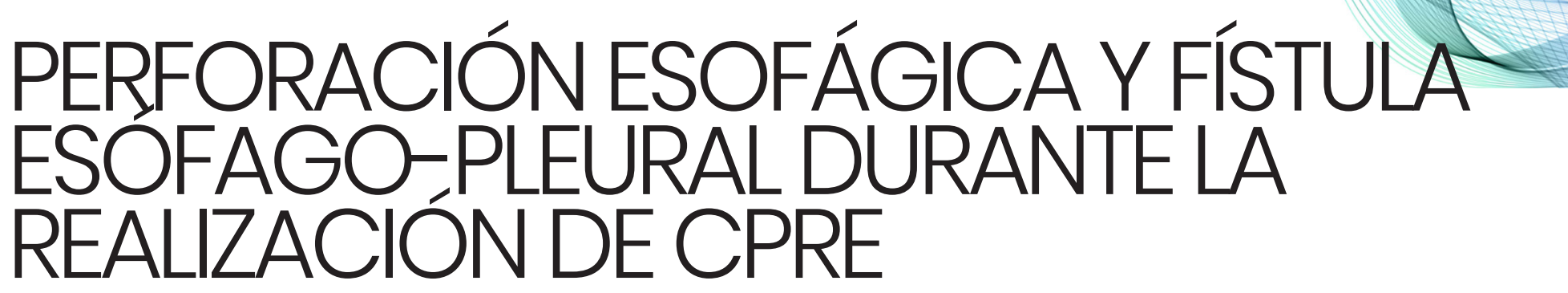

\title{
ESOPHAGICAL PERFORATION AND ESOPHAGO- PLEURAL FISTULA DURING THE PERFORMANCE OF ERCP
}

\section{Martínez Huertas C', Escobedo Araque C', Milena Muñoz A², Sánchez Talavera S'}

'Complejo Hospitalario de Jaén. Jaén.

${ }^{2}$ Hospital Universitario San Cecilio. Granada.

\section{Resumen}

Las perforaciones iatrogénicas del tracto gastrointestinal secundarias a procedimientos endoscópicos diagnósticos o terapeúticos son complicaciones raras pero severas, con una elevada morbimortalidad. Asuvez la fístula-esófago pleural es una complicación muy rara, que normalmente se produce tras una neumectomía o evento traumático y en menor medida asociada a patología infecciosa o neoplásica; Su gravedad depende principalmente de la coexistencia de procesos sépticos intraabdominales y del volumen de secreción. Se acompaña de infecciones serias y potencialmente mortales, de ahí la importancia de hacer un diagnostico precoz. El diagnóstico se realiza habitualmente mediante $\mathrm{TC}$, preferiblemente con contraste oral para visualizar el trayecto fistuloso. Aunque la endoscopia puede ayudar no sólo en el diagnóstico, sino también en su tratamiento, en gran parte de los casos el tratamiento de elección es la cirugía.

Palabras clave: perforación esofágica, fístula esófagopleural, endoscopia, TC.

\section{CORRESPONDENCIA}

Carmen Martinez Huertas

Complejo Hospitalario de Jaén

23007 Jaén

cmhuertas@hotmail.com

\section{Abstract}

latrogenic perforations of the gastrointestinal tract related to diagnostic or therapeutic endoscopy are a rare but severe adverse event, associated with significant morbidity and mortality. Esophagopleural fistula (EPF) is a rare postoperative complication that commonly occurs after pneumonectomy, or after other traumatic events, and less commonly associated with infectious or neoplasic pathology. Esophageal fistula could be congenital, postsurgical, traumatic, infectious or neoplasic. Their severity depends on the coexistence of septic intraabdominal process and the debit of the fistula. EPF is usually accompanied by serious infection and lifethreatening morbidity, that underlines the importance of an early diagnosis. The main diagnosis test is the $\mathrm{CT}$, if possible, with oral contrast in order to see the fistulous path. Although endoscopy can help not only in diagnosis but also in the treatment, in most cases the treatment of choice is the surgery.

Keywords: esophageal perforation, esophago-pleural fistula, endoscopy, CT.

\section{Introducción}

La CPRE es un procedimiento bien conocido con utilidad diagnóstica y terapéutica en el manejo de problemas pancreáticobiliares y se considera seguro y efectivo incluso en población de edad avanzada1. Las complicaciones más frecuentes son las pancreatitis, colangitis, hemorragias, y como en todo procedimiento endoscópico la perforación $n^{1,2,3}$. 
La perforación iatrogénica durante un procedimiento endoscópico se define como la presencia de gas o contenido luminal fuera del tracto gastrointestinal ${ }^{4}$; muchas se detectan durante el procedimiento ${ }^{2}$ pero pueden pasar desapercibidas3. En el caso de perforaciones esofágicas iatrogénicas, la mayoría se asocian con procedimientos terapéuticos ${ }^{4}$ y son más frecuentes en esófago distal5. El riesgo varía en función de las características del paciente y habilidades del endoscopista ${ }^{1}$. y el tiempo de diagnóstico es fundamental, pues la fuga de contenido alimenticio o líquido a cavidad torácica o abdominal puede producir una infección fatal².

Las fístulas esofágicas pueden ser de etiología congénita, postquirúrgica, traumáticas, infecciosa o neoplásica. Etiopatogénicamente resultan de dehiscencias de suturas, daño tisular secundario a traumatismo, isquemia, infección o infiltración tumoral. Generalmente se abren a tráquea o bronquios, y mucho menos frecuentemente al mediastino o como en nuestro caso a la pleura6. Las fístulas posquirúrgicas del tracto digestivo superior son complicaciones graves, y presentan una elevada morbimortalidad; Su gravedad depende en gran medida de la coexistencia de procesos sépticos intraabdominales y del volumen de secreción, y su tratamiento es complejo ${ }^{6}$.

Un diagnóstico precoz ha demostrado tener un impacto sustancial en el tratamiento y sus resultados ${ }^{4}$. La presencia de líquido y aire extraluminal en TC se asocia con una perforación significativa de la pared intestinal y conllevan peor pronóstico ${ }^{3}$. Ante la presencia de síntomas y signos de sospecha de perforación iatrogénica tras un procedimiento endoscópico (dolor abdominal anormal, dolor torácico, disnea ${ }^{4}$,taquicardia, fiebre, leucocitosis $\left.{ }^{1} . ..\right)$ debe realizarse un TC de forma precoz para evitar retrasos en el diagnóstico ${ }^{4}$. La TC permite detectar pequeñas cantidades de gas en peritoneo, retroperitoneo y mediastino, así como líquido libre, colecciones, neumotórax y empiema, y aporta buena definición de estructuras adyacentes ${ }^{4}$. Se recomienda la utilización de contraste oral hidrosoluble que permita visualizar con precisión el trayecto fistuloso ${ }^{6}$.

El tratamiento es controvertido, No existe evidencia basada en las guías para el manejo de las perforaciones endoscópicas iatrogénicas ${ }^{2,3}$. En líneas generales se puede decir que los factores que favorecen el éxito de un tratamiento endoscópico son el reconocimiento y tratamiento precoz de la misma (<24horas), tamaño de la perforación $(<2 \mathrm{~cm})$, ausencia de paso de contenido intraluminal al mediastino, ausencia de comorbilidades y de inestabilidad clínica del paciente, tratamiento por un endoscopista experto y manejo por un equipo multidisciplinar con experiencia ${ }^{1}$. En casos de inestabilidad clínica, reconocimiento tardío $(>24 \mathrm{~h})$, presencia de colecciones líquidas en mediastino y/o cavidad pleural, o comorbilidades estaría indicada la cirugía ${ }^{4}$.

\section{Caso Clínico}

Presentamos el caso de un paciente de 89 años con antecedente de hipertensión arterial y enfermedad de Crohn que acudió a urgencias por un episodio de dolor abdominal de varios días de evolución centrado en epigastrio. En la analítica se observó un aumento de la Bilirrubina total, transaminasas y PCR.
Se realizó ecografía abdominal que mostró colelitiasis sin signos de colecistitis e importante dilatación de la vía biliar intra y extrahepática con una litiasis en tercio distal del colédoco.

Se procedió a realizar CPRE, objetivando durante la realización de la misma un desgarro en el tercio distal del esófago sin poder descartar perforación secundaria, motivo por el cual se suspendió el procedimiento y se solicitó TC para completar estudio.

La TC con contrastes oral e intravenoso mostró la ya conocida dilatación de la vía biliar con una litiasis de $16 \mathrm{~mm}$ en colédoco distal como causa de la misma (Figura 1) y un neumotórax inferior izquierdo (Figura 2), así como una solución de continuidad de la pared izquierda del esófago distal, junto a la unión esófago-

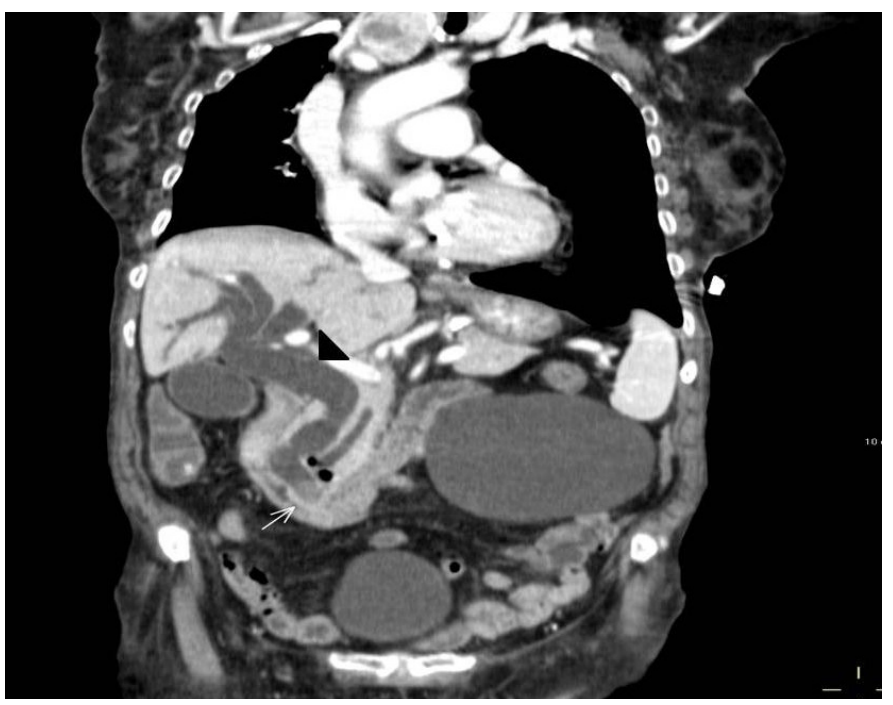

Figura 1

Reconstrucción coronal de TC abdominal con contrastes oral e intravenoso. Marcada dilatación de la vía biliar intra y extrahepática (cabeza de flecha) hasta colédoco distal, donde se observa imagen redondeada discretamente hiperdensa en relación con coledocolitiasis (flecha blanca).

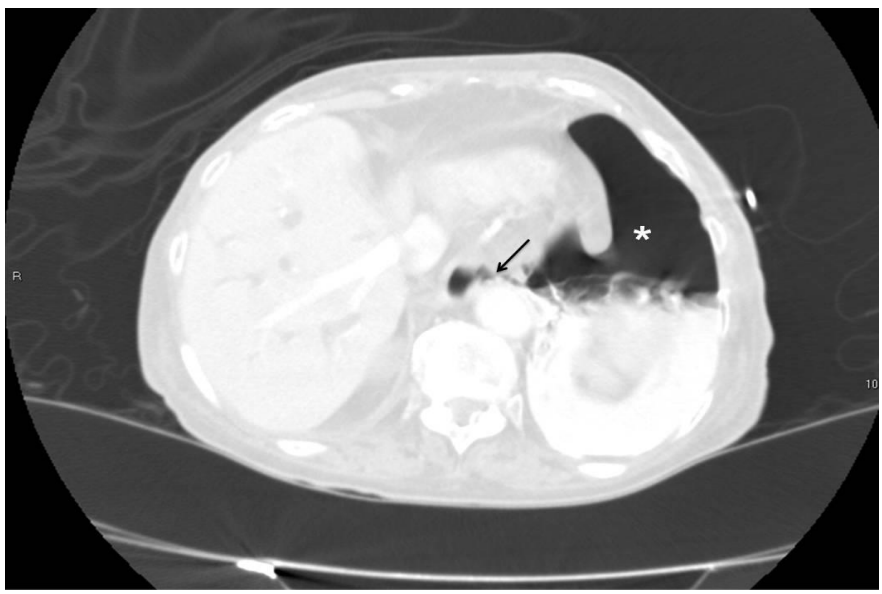

Figura 2

Corte axial de TC en ventana de pulmón. Solución de continuidad de la pared izquierda del esófago distal (flecha negra) con neumotorax anteroinferior izquierdo $\left({ }^{*}\right)$. 
gástrica, con extravasación de contraste oral al espacio pleural (Figuras 1 y 2); confirmando dichos hallazgos la sospecha de perforación esofágica con una fístula esófago-pleural asociada.

Posteriormente el paciente fue intervenido quirúrgicamente.

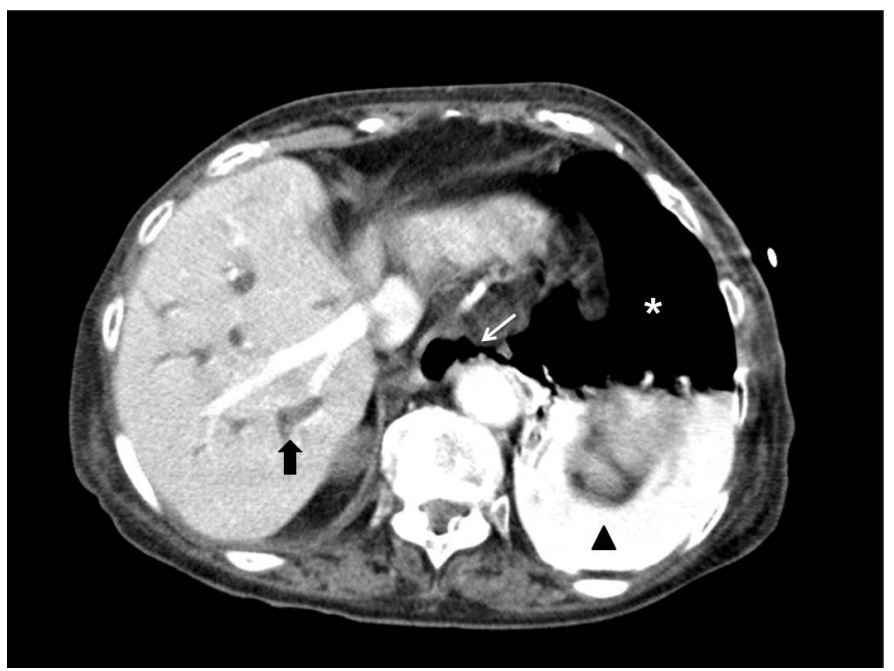

\section{Figura 3}

Corte axial de TC con contrastes oral e intravenoso. Solución de continuidad de la pared izquierda del esófago distal (flecha blanca), con fuga de contraste oral al espacio pleural (cabeza de flecha) y neumotorax anteroinferior izquierdo $\left({ }^{*}\right)$. Dilatación de la vía biliar intrahepática (flecha negra).

\section{Discusión}

Las fístulas posquirúrgicas del tracto digestivo superior son complicaciones graves y potencialmente mortales ${ }^{3}$ y el tiempo de diagnóstico es crítico para el manejo y desenlace del paciente ${ }^{4}$. Ante la presencia de síntomas y signos de sospecha debe realizarse un TC de forma precoz ${ }^{4}$, utilizando contraste oral ${ }^{6}$. En el caso de que endoscópicamente se detecte la perforación se recomienda describir con detalle el tamaño y la localización y considerar el tratamiento endoscópico en caso de que sea posible ${ }^{4}$, teniendo en cuenta que los factores que se relacionan con el éxito del tratamiento endoscópico son el reconocimiento y tratamiento precoz de la misma, el tamaño, la ausencia de fuga de contenido intraluminal al mediastino, la ausencia de comorbilidades y de inestabilidad clínica del paciente, así como que el tratamiento se realice por un endoscopista experto y el manejo por un equipo multidisciplinar con experiencia, siendo siempre necesaria una evaluación individualizada del paciente ${ }^{4}$.

\section{Bibliografía}

1. Kemmian D.Johnson, Abhilash Perisetti, Benjamin Tharian, Ragesh Thandassery, Priya Jamidar,Hemant Goyal, Sumant Inamdar. Endoscopic Retrograde Cholangiopancreatography-Related Complications and Their Management Strategies: A “Scoping” Literature Review. Dig Dis Sciences. 2019 (12); 1-15

2. Dae Hwan Kang, Dae Gon Ryu, Cheol Woong Choi, Hyung Wook Kim, Su Bum Park, Su Jin Kim and Hyeong Seok Nam. Clinical outcomes of iatrogenic upper gastrointestinal endoscopic perforation: a 10-year study. BMC Gastroenterology. 2019; 19: 218

3. Jhon-Han Wu, Hong-Ming Tsai, Chiung-Yu Chen, Yao-Sheng Wang. Computed tomography classification of endoscopic retrograde cholangiopancreatographyrelated perforation. J Med Sci. 2019;1-6.

4. Gregorios A. Paspatis, Jean-Marc Dumonceau, Marc Barthet, Søren Meisner, Alessandro Repici, Brian P. Saunders, Antonios Vezakis, Jean Michel Gonzalez, Stine Ydegaard Turino, Zacharias P. Tsiamoulos, Paul Fockens Cesare Hassan. Diagnosis and management of iatrogenic endoscopic perforations: European Society of Gastrointestinal Endoscopy (ESGE) Position Statement. Endoscopy 2014;46(8):693-71

5. Harpreet K. Pannu, Elliot K. Fishman. Complications of Endoscopic Retrograde Cholangiopancreatography: Spectrum of Abnormalities Demonstrated with CT. RadioGraphics 2001; 21:1441-1453.

6. L.R Rábago, JL. Castro, D. Joya, N Herrera, F.Gea, P. Mora, C.Blesa. Endoscopic closure of tracheo-esophageal perforation and postoperative upper gastrointestinal fistulae with Tissucol. Gastroenterología y hepatología $2000 ; 23(2): 82-86$ 